\title{
Mini Review: Nano-Technology based Drug Deliveries
}

\author{
Kuilong Wang \\ School of pharmacy, Nanjing University of Chinese Medicine, Nanjing 210023, P. R. China
}

\section{ABSTRACT}

Targeted drug delivery with nano-technology has been researched and identified as being efficient across many treatment conditions. This review assesses some of the existing research work and evidence practice in using nano-technology based drug carriers.

Keywords: Nano-technology, drug delivery, nanoparticle formulation, nano-technology carriers, drug resistance

Article Info: Received 03 Oct, 2018; Review Completed 09 Nov 2018; Accepted 12 Nov 2018; Available online 15 Nov 2018

Cite this article as:

Wang K, Mini Review: Nano-Technology based Drug Deliveries, Journal of Drug Delivery and Therapeutics. 2018; 8(6):268-271 DOI: http://dx.doi.org/10.22270/jddt.v8i6.2053

\section{Introduction}

Targeted drug delivery or smart delivery is treatment methods that are used for the purpose of increasing medicine delivery and treatment efficacy ${ }^{1-3}$. Passive and active drug delivery methods are used with drug delivery vehicles that are even capable of crossing the blood-brain barrier $^{4-8}$. This paper reviews the different Nanotechnology based drug carrier or drug delivery systems that have been implemented and tested in contemporary practices. The pros and differentiated aspects of such delivery systems as compared to others are discussed.

\section{Nano-technology forms of drug delivery}

Nano-technology based forms of drug delivery are one of the most popular methods of drug delivery. Nanoparticles are smaller in size and this allows for better delivery of drugs that do not dissolve in water and thus can complete the first pass through liver metabolism ${ }^{9-13}$. Since nanotechnology forms of drug delivery ensure that the drug is circulated in the body for a longer time, it means that the drug is functional for more time. Plasma levels are therefore less-fluctuating and more stable. Nanotechnology form of methods is useful against drug resistance effects. Nano-delivery methods can even cross the blood-brain barrier with least cytotoxicity ${ }^{14-17}$. These drug delivery methods are further extended as observed in the case of Yang et al.'s research work where gene delivery to cancer cells is facilitated. The delivery of nanoparticles through the blood-brain barriers as presented in Kang et al.,10 research is presented below.

Yung et al., research focused on the efficient delivery of the AM-21 also called the microRNA-21 which is used in human cancer treatment. The authors propose the use of lipid nanoparticle formulations called the QTsome ${ }^{18-23}$. Nano-technology form of drug delivery was considered more effective in this situation. AM-21 is an effective therapeutic agent, but then after its release into the bloodstream, AM 21 is also cleared in a very fast manner from the bloodstream. Additionally, the medicine is not capable of penetrating the cellular membrane and hence does not deliver medicine to targeted sites efficiently. In the past, a chemical modification of the lipid nanoparticle backbone has been considered necessary $24-27$. The use of Nano-carriers in such situations showed enhanced therapeutic effects and reduced the cytotoxicity in normal cells. Off-target cytotoxicity was reduced to a great extent because of active targeting. Use of combination and Nano delivery methods reduces tumor growth more than others as highlighted below. 


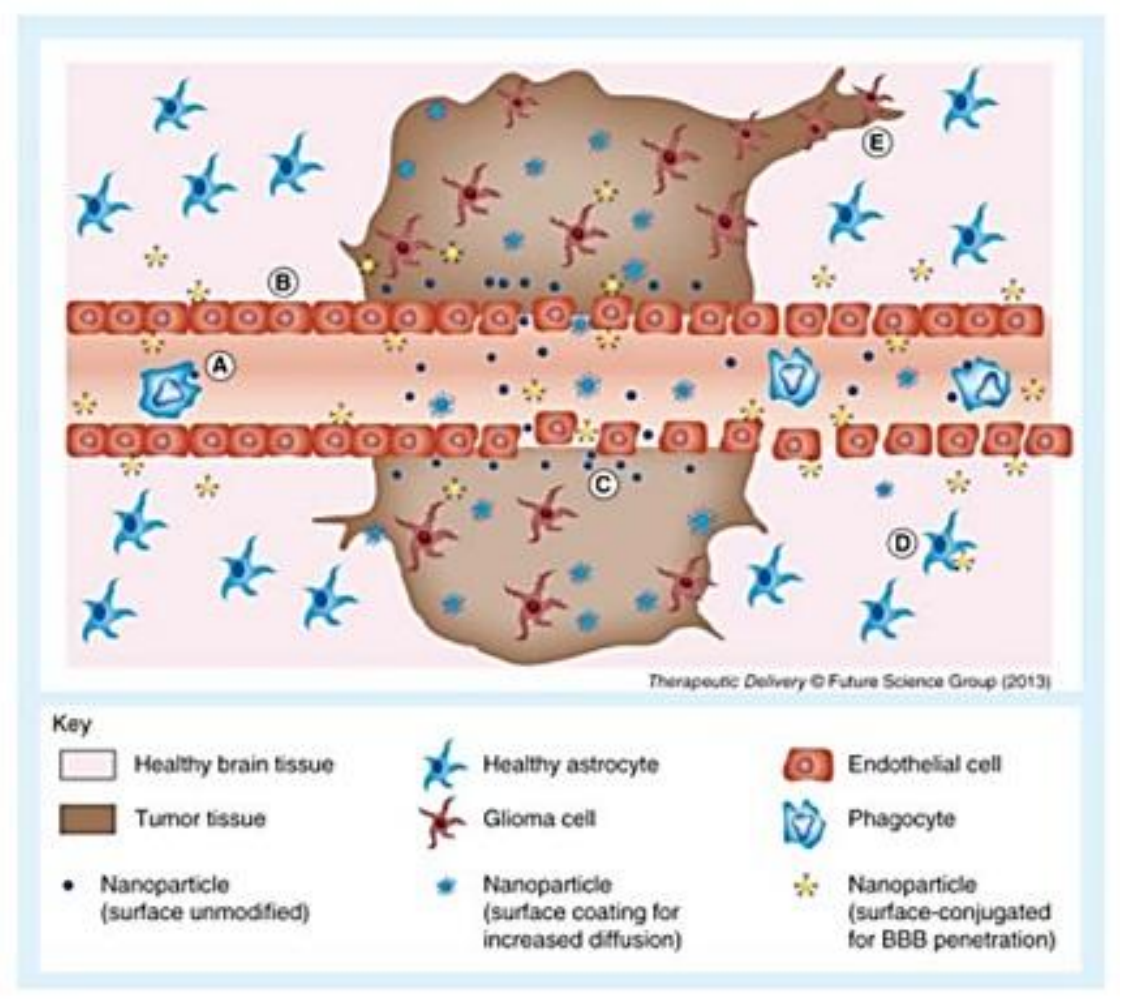

Figure 1: Nanoparticles and the BBB, (Kang et al.,)

A

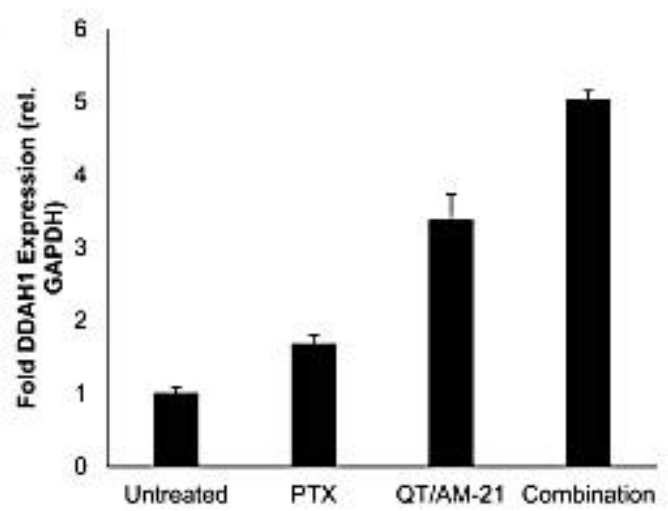

B

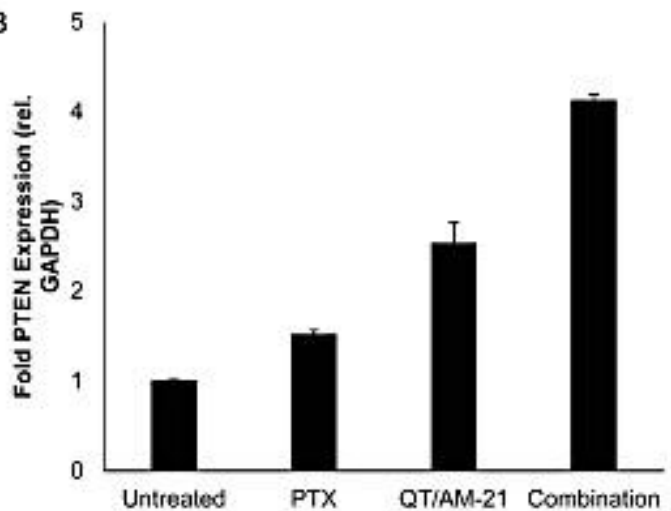

Figure 2: In-vivo results (Yung et al.,)

The use of the QTsome as a novel nanoparticle formulation ensures that a more responsive profile is developed for medicine delivery28-30. Nanoparticle-based platforms for delivery are an enhanced system on account of its low molecular weight. The small size and high drug loading capacity of QTsome nanoparticle lend to better long-term stability.

\section{Targeted delivery}

Targeted delivery helps deliver the needed medicines to the sites of action. Camptothecin acts as a DNA topoisomerase 1 inhibitor. It is used as an anti-tumor agent. Replication of cancer DNA is terminated with the Camptothecin. Although exhibiting cytotoxicity against tumor cells, the E-lactone ring in the drug Camptothecin is unstable. As a result of its instability, inactive carboxylates are formed. Moreover, the drug has poor aqueous solubility ${ }^{31-34}$. Issues in biodistribution exist as well. In this context, targeted delivery systems such as the nanotechnology systems by property of selective deliverance will send the drugs directly into the tumor cell, instead of distributing it across normal tissues. Permeability and retention effects cause the delivery of nanoparticles across blood vessels as well. Compared to the traditional delivery modes, the medicine is retained for a much longer time.

The work of Kang et al. on the delivery of nanoparticles in the case of brain tumor highlights how chemotherapy results in drug resistance issues and issues of cytotoxicity in off-target regions for brain tumors like the glioblastoma. Brain tumors cannot be detected at an early stage, and when detected, the usual course of treatment is surgery followed by chemotherapy or radiation. Chemotherapeutic drugs usually end up causing high levels of off-target cytotoxicity, and over time, patients could end up becoming resistance to some chemotherapeutic drugs as well. This drug resistance questions the efficacy of the treatment program for the patient. The efficacy of treatment becomes questionable. The chemotherapeutic drugs fail to cross through the blood-brain barrier (BBB) and the insufficient concentrations will not be enough to restrict the progressive growth of tumors in tumor sites even after debulking surgeries ${ }^{35-39}$. In this context, the 
nanotechnology form of drug delivery crosses the barrier and delivers medicine at the tumor site. It is used in antipsychotic drug delivery. Psychosis is a severe mental health problem involving delusions, catatonia and more. Antipsychotics that are used for their treatment have many side effects. Sun et al. argue for the use of nanoparticles in antipsychotics administration. Brain targeting drugs help create slow release profiles of drugs that are far more aqueous soluble and efficient than other delivery forms.

Nanotechnology can be used for the early detection of the tumor in some situations and its value is extended beyond just a carrier as observed in current research works. For example, consider the research work of Yang et al. where nanotechnology is applied to use nanoscaled exosome mimics NM. Exosomes are the smallest of the extracellular

\section{References}

1. Kang, C., Qin, J., Osei, W. \& Hu, K. Age-dependent Mitochondrial Targeting Of Protein Kinase C Epsilon In Cardioprotection. The FASEB Journal (2017).

2. Han, R., Sun, Y., Kang, C., Sun, H. \& Wei, W. Amphiphilic dendritic nanomicelle-mediated co-delivery of 5-fluorouracil and doxorubicin for enhanced therapeutic efficacy. Journal of Drug Targeting 2017; 25:140-148.

3. Duan, Y., et al. Bioactivity evaluation-based ultra highperformance liquid chromatography coupled with electrospray ionization tandem quadrupole-time-of-flight mass spectrometry and novel distinction of multisubchemome compatibility recognition strategy with Astragali Radix-Fructus Corni herb-pair as a case study. $J$ Pharm Biomed Anal 2016; 129:514-534.

4. Sun, Y., et al. Co-delivery of dual-drugs with nanoparticle to overcome multidrug resistance. European Journal of BioMedical Research 2016; 2:12-18.

5. Ai, R., et al. Comprehensive epigenetic landscape of rheumatoid arthritis fibroblast-like synoviocytes. Nat Commun 2018; 9:1921.

6. Fan, S., et al. Computationally expanding infinium HumanMethylation450 BeadChip array data to reveal distinct DNA methylation patterns of rheumatoid arthritis. Bioinformatics 2016; 32:1773-1778.

7. Liu, F., Sun, Y. \& Kang, C. Controlling Amphiphilic Functional Block Copolymers' Self-Assembly: From Structure to Size. (2016).

8. Song, L., et al. Crocetin inhibits lipopolysaccharide-induced inflammatory response in human umbilical vein endothelial cells. Cellular Physiology and Biochemistry 2016; 40:443-452.

9. Sun, Y., Kang, C., Liu, F. \& Song, L. Delivery of antipsychotics with nanoparticles. Drug Development Research 2016; 77:393-399.

10. Kang, C., et al. Delivery of nanoparticles for treatment of brain tumor. Current Drug Metabolism 2016; 17:745-754

11. Xue, X., et al. Discovery of novel inhibitors disrupting HIF$1 \alpha /$ von Hippel-Lindau interaction through shape-based screening and cascade docking. PeerJ 2016; 4:e2757.

12. Hersch, S.J., et al. Divergent protein motifs direct elongation factor P-mediated translational regulation in Salmonella enterica and Escherichia coli. MBio 2013; 4:e00180-00113.

13. Shuhong, X., et al. Dynamic expression of AQP4 in early stageof ischemia/reperfusion rats and cerebral edema. Chinese Pharmacological Bulletin 2016; 32:1433-1441.

14. Peng, J., et al. Enhanced Liver Regeneration After Partial Hepatectomy in Sterol Regulatory Element-Binding Protein (SREBP)-1c-Null Mice is Associated with Increased vehicles and contain genetic information for intercellular communication. The rapid abnormal proliferation of cells as in the case of cancer can be handled by communication of genetic and proteomic information through the nanotechnology applied exosome mimics. Both in-vitro and invivo analysis holds many benefits ${ }^{10}$.

\section{Conclusion}

Targeted delivery methods ensure that drugs are delivered to the required site of action in an efficient manner. The work reviewed the Nano-technology drug delivery systems such as AM-21, Camptothecin, chemotherapeutic drugs for the brain tumor and antipsychotic drugs for psychosis. The targeted delivery does not only increase efficacy but also causes reduced harmful actions in target off-site.

Hepatocellular Cholesterol Availability. Cellular Physiology and Biochemistry 2018; 47:784-799.

15. Yang, Z., et al. Functional exosome-mimic for delivery of siRNA to cancer: in vitro and in vivo evaluation. Journal of Controlled Release 2016; 243:160-171.

16. Kang, C., Hernandez, V.A. \& Hu, K. Functional interaction of the two-pore domain potassium channel TASK-1 and caveolin-3. Biochimica et Biophysica Acta (BBA)-Molecular Cell Research 2017; 1864:537-1544.

17. Waller, A.P., et al. GLUT12 functions as a basal and insulinindependent glucose transporter in the heart. Biochimica et Biophysica Acta (BBA)-Molecular Basis of Disease 2013; 1832:121-127.

18. Li, Q., et al. Identification by shape-based virtual screening and evaluation of new tyrosinase inhibitors. PeerJ 2018; 6:e4206.

19. Chen, Y., et al. Identification of 4 -aminoquinoline core for the design of new cholinesterase inhibitors. PeerJ 2016; 4:e2140.

20. Kang, C. \& Hu, K. Impact of hypoxia in the expression and regulation of the TASK-1 potassium channel in cardiac myocytes. The FASEB Journal 2016; 30:lb598-lb598.

21. Kang, C. Ion channels, protein kinase $C$ and caveolae in cardioprotection, (The Ohio State University, 2015).

22. Yung, B.C., et al. Lipid nanoparticles composed of quaternary amine-tertiary amine cationic lipid combination (QTsome) for therapeutic delivery of AntimiR-21 for lung cancer. Molecular pharmaceutics 2016; 13:653-662.

23. Cheng, X., et al. Lipid Nanoparticles Loaded with an Antisense Oligonucleotide Gapmer Against Bcl-2 for Treatment of Lung Cancer. Pharmaceutical research 2017; 34:310-320.

24. Fan, S. \& Chi, W. Methods for genome-wide DNA methylation analysis in human cancer. Brief Funct Genomics 2016; 15:432-442.

25. Kang, C. \& Hu, K. Modulation of the two-pore domain potassium channel TASK-1 by caveolin-3. The FASEB Journal 2015; 29:845.814.

26. Kang, C., Sun, Y., Wang, M. \& Cheng, X. Nanosized camptothecin conjugates for single and combined drug delivery. European Journal of BioMedical Research 2016; 2:814.

27. Qiao, H., et al. Orally delivered polycurcumin responsive to bacterial reduction for targeted therapy of inflammatory bowel disease. Drug Delivery 2017; 24:233-242.

28. Liu, F., Sun, Y., Kang, C. \& Zhu, H. Pegylated Drug Delivery Systems: From Design to Biomedical Applications. Nano LIFE 2016; 6:1642002. 
29. Sun, Y., Kang, C., Yao, Z., Liu, F. \& Zhou, Y. Peptide-Based Ligand for Active Delivery of Liposomal Doxorubicin. Nano Life 2016; 6:1642004.

30. Fan, S., Huang, K., Ai, R., Wang, M. \& Wang, W. Predicting CpG methylation levels by integrating Infinium HumanMethylation450 BeadChip array data. Genomics 2016; 107:132-137.

31. Qiao, H., et al. Redox-triggered mitoxantrone prodrug micelles for overcoming multidrug-resistant breast cancer. Journal of drug targeting 2018; 26:75-85.

32. Kang, C., Qin, J., Osei, W. \& Hu, K. Regulation of protein kinase $\mathrm{C}$-epsilon and its age-dependence. Biochemical and Biophysical Research Communications 2017; 482:1201-1206.

33. Sun, Y., et al. RGD Peptide-Based Target Drug Delivery of Doxorubicin Nanomedicine. Drug development research 2017; 78:283-291.
34. Kang, C. \& Hu, K. Role of caveolin-3 in adenosine-induced increase in mitochondrial PKCE. The FASEB Journal 2013; 27:1191.1197-1191.1197.

35. Cheng, X. \& Lee, R.J. The role of helper lipids in lipid nanoparticles (LNPs) designed for oligonucleotide delivery. Adv Drug Deliv Rev 99, 129-137 (2016).

36. Sun, Y. \& Kang, C. Self-Assembly of Peptides into Hydrogel. Journal of Organic \& Inorganic Chemistry 2016; 2:5.

37. Yao, Z., Sun, Y. \& Kang, C. Structure and self-assembly of multicolored Naphthalene Diimides Semiconductor. Nano LIFE 2016; 6:1642007.

38. Cheng, X., et al. T7 Peptide-Conjugated Lipid Nanoparticles for Dual Modulation of Bcl-2 and Akt-1 in Lung and Cervical Carcinomas. Molecular pharmaceutics 2018; 15:4722-4732.

39. Zhong, X., Sun, Y., Kang, C. \& Wan, G. The theory of dielectrophoresis and its applications on medical and materials research. European Journal of BioMedical Research 2017; 2:7-11. 